\title{
Axillary hematoma secondary to arterial pseudoaneurysm following prophylactic mastectomy: A case report
}

\author{
Meghana R.K. Helder, Jane Case, Marilyn Morton, Sandhya Pruthi, Gustavo Oderich, Amy C. Degnim* \\ Departments of Surgery, Radiology, and Internal Medicine, Mayo Clinic, Rochester, MN, United States
}

Received: February 10, 2016

DOI: $10.5430 /$ css.v2n2p30

\author{
Accepted: March 3, 2016 \\ Online Published: March 10, 2016 \\ URL: http://dx.doi.org/10.5430/css.v2n2p30
}

\begin{abstract}
Recurrent axillary hematomas after prophylactic mastectomy are rare. We present one such case of a 62-year-old female with a recurrent axillary hematoma secondary to an arterial pseudoaneurysm. This report also details our treatment plan along with a review of the relevant literature. To our knowledge, this is the first case report to document a pseudoaneurysm of the axilla after mastectomy.
\end{abstract}

Key Words: Pseudoaneurysm, Bleeding, Axillary dissection, Axillary hematoma, Surgical complications

\section{INTRODUCTION}

Although mastectomy-bed hematomas are well-known complications, occurring in approximately $2 \%-10 \%$ of patients, ${ }^{[1-3]}$ axillary hematomas are rare. ${ }^{[4,5]}$ In most cases, there is no identifiable source of bleeding. Recurrent axillary hematomas after operation have not been reported to our knowledge. We present a case of recurrent axillary hematoma after prophylactic mastectomy secondary to a pseudoaneurysm of an axillary arterial branch.

\section{CASE REPORT}

A 62-year-old female presented to an outside institution where she underwent a bilateral mastectomy with left sided sentinel lymph node biopsy for biopsy proven ductal carcinoma in situ in the left breast. Her medical history was significant for hypertrophic cardiomyopathy with pacemaker, paroxysmal atrial fibrillation, history of a transient ischemic attack, and chronic warfarin anticoagulation. Her immediate postoperative course was complicated by a right-sided axillary hematoma on postoperative day two, despite normal preoperative coagulation studies and discontinuation of oral anticoagulation. At operation, $500 \mathrm{ml}$ of hematoma was evacuated and small bleeders were ligated. Specifically, one area near the latissimus dorsi was ligated with suture, but no causative bleeding vessel was identified. After 30 minutes of observation and satisfaction with hemostasis, the wound was closed with a new drain in place. Her anticoagulation was restarted three days post-operatively.

The patient had recurrent bleeding and hematoma 6 weeks after the first reoperation, at which time she underwent a second surgical exploration and evacuation of the hematoma through the same mastectomy scar. Approximately one liter of blood was evacuated and red blood cell transfusion was required. Per the outside operative note, no surgical bleed-

\footnotetext{
*Correspondence: Amy C. Degnim; Email: degnim.amy@mayo.edu; Address: Division of General Surgery, Mayo Building 12, Mayo Clinic, 200
} First St SW, Rochester, MN 55905, United States . 
ing was identified, and the wound was closed after placing a new drain. Her anticoagulation was resumed five days postoperatively. On postoperative day eight, the patient experienced leakage around the drain, suggesting obstruction of the drainage tube. The drain was removed eleven days after the second hematoma evacuation. However, the patient noted a palpable tender lump in the axilla one week later. Subsequently over one month, she underwent two weekly aspirations of bloody fluid from what was described as a seroma, with $40 \mathrm{cc}$ on the second occasion. International normalized ratio (INR) at that time was noted to be 3.6. The patient described that the repeated aspirations did not resolve the palpable mass and was referred to our center for further evaluation and treatment.

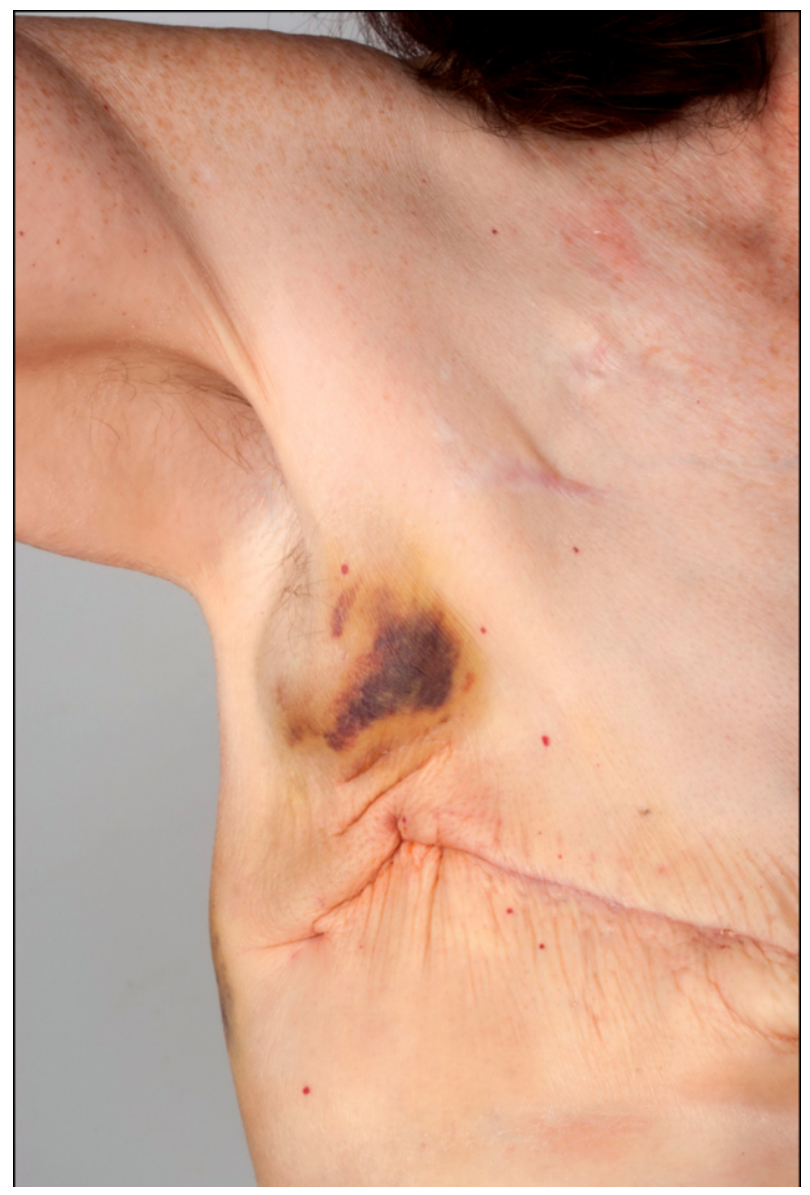

Figure 1. Patient's right axillary hematoma with ecchymosis on presentation to our clinic

Physical examination revealed a $6 \mathrm{~cm} \times 8 \mathrm{~cm}$ wellcircumscribed tense mass in the low right axilla with associated focal tenderness and overlying moderate ecchymosis (see Figure 1). There was no evidence of infection or cellulitis. Range of motion was restricted at the right shoulder due to discomfort. Diagnostic axillary ultrasound was obtained and demonstrated a $5.7 \mathrm{~cm}$ hypoechoic solid appearing mass Published by Sciedu Press with localized arterial flow within the superior and medial portions of the mass. This was consistent with a partially thrombosed pseudoaneurysm that appeared to arise from a three $\mathrm{mm}$ artery (see Figure 2). Initial management entailed thrombin injection directed at the neck of the feeding arterial vessel and this resulted in thrombosis. CT angiogram was obtained the next day to define the anatomy of the pseudoaneurysm in relation to the main axillary artery. This demonstrated no evidence for active contrast extravasation, with the pseudoaneurysm arising from a small branch of the axillary artery a distance of $5 \mathrm{~cm}$ from the main artery (see Figure 3).

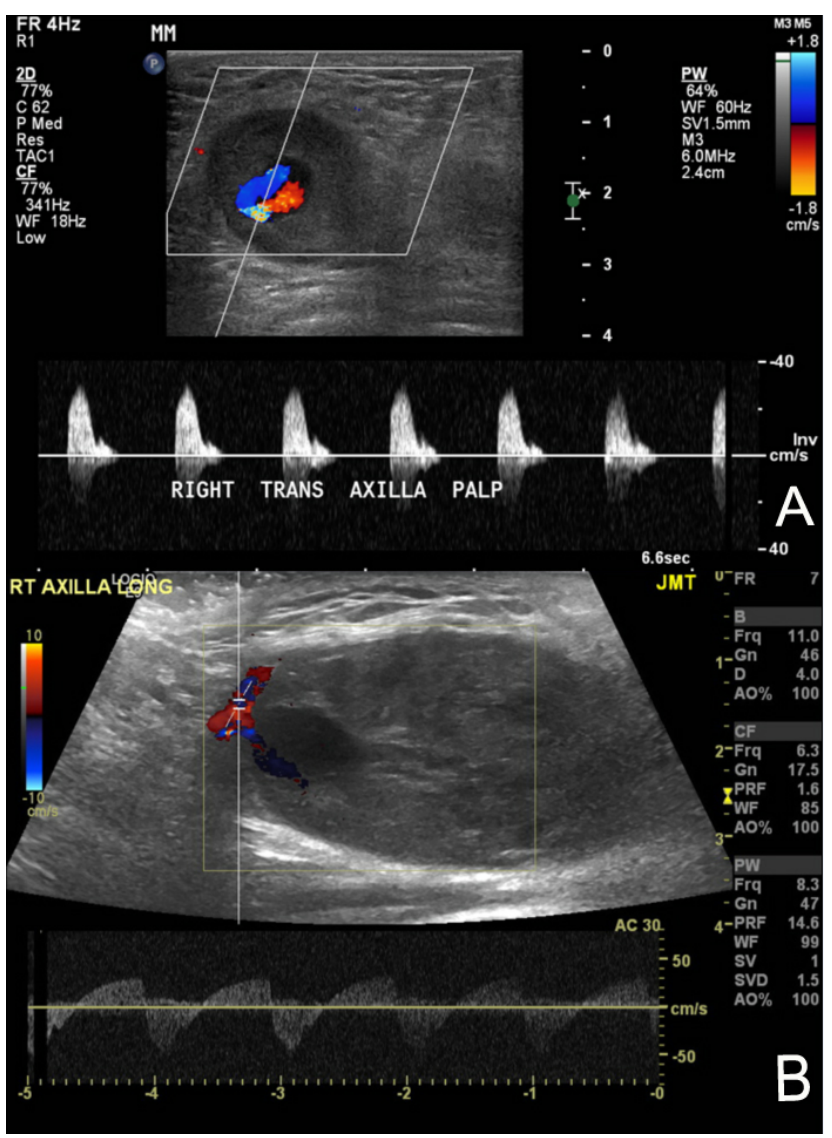

Figure 2. Spectral Doppler image of the palpable right axillary mass

(A) Transverse color and spectral Doppler image of the palpable right axillary mass shows a $2.5 \mathrm{~cm}$ hypoechoic mass with central swirling (red/blue) flow. This "yin-yang" appearance is typical for a pseudoaneurysmal sac; (B) Longitudinal color and spectral

Doppler image of periphery of the right axillary mass shows a small adjacent artery with bidirectional flow. This is characteristic of the feeding artery of a pseudoaneurysm

Her anticoagulation was held, and one week later with a normal INR, she was taken to the operating room for hematoma evacuation and definitive management of the pseu- 
doaneurysm. A transverse incision was made at the inferior axillary hairline, located over the superior aspect of the palpable mass. Approximately $35 \mathrm{cc}$ of organized hematoma was evacuated and the pseudoaneurysm capsule was excised Pulsatile bleeding was immediately apparent from the $2 \mathrm{~mm}$ feeding vessel at the superior aspect of the pseudoaneurysm (see Figure 4), and this was ligated. Additional small bleeding sites were cauterized or ligated and a drain was placed. The subcutaneous tissues were quilted down to the chest wall and the wound was closed in two layers.
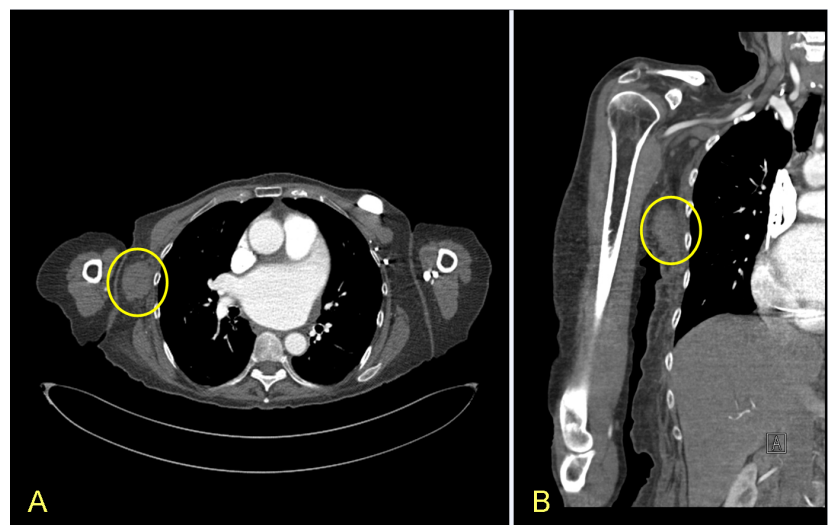

Figure 3. Transverse and Longitudinal images from CT angiogram

Transverse (A) and Longitudinal (B) images from CT angiogram performed the day after thrombin injection show a $3.5 \mathrm{~cm}$ avascular soft tissue mass (yellow circles) in the low right axilla abutting the chest wall consistent with thrombosed pseudoaneurysm. There is no evidence of contrast extravasation or residual pseudoaneurysm formation

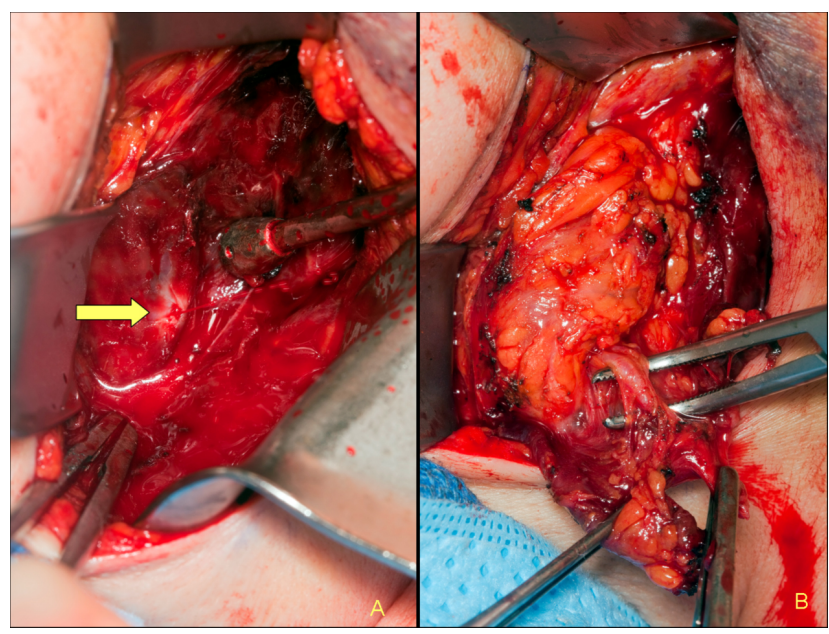

Figure 4. Intraoperative picture and further dissectionof the ruptured arterial pseudoaneurysm

(A) Intraoperative picture of the ruptured arterial pseudoaneurysm with pulsatile flow (arrow); (B) Further dissection revealed the feeding arterial branch of the pseudoaneurysm
The patient was seen in follow up on postoperative day 4 with no evidence of hematoma or infection. The drain had $5 \mathrm{cc}-10 \mathrm{cc}$ of sero-sanguineous fluid from the last 12 hours at which point her anticoagulation was restarted. She was seen again on postoperative day seven at which time the drain was removed.

\section{Discussion}

A pseudoaneurysm is a contained hematoma that results from disruption of the arterial wall. ${ }^{[6]}$ The most feared complication is rupture. It is most frequent in elderly patients, in those patients with known atherosclerosis, and patients on anticoagulation therapy. ${ }^{[7]}$ Most pseudoaneurysms are secondary to either trauma or are iatrogenic. Iatrogenic pseudoaneurysm of the femoral artery has been described after cardiac catheterization at a rate of $1.6 \%{ }^{[6]}$ There are reports of superior thyroid artery pseudoaneurysms after chemical parathyroidectomy, radiotherapy, and fine needle aspiration biopsy. ${ }^{[6]}$ Pseudoaneurysms have also been reported to occur after core needle biopsy of a breast lesion. ${ }^{[8]}$ However, in most cases these pseudoaneurysms are self-limited or treated with compression, thrombin injection, or alcohol injection with excellent results. ${ }^{[9]}$ For larger vessel pseudoaneurysms, endovascular repair and covered stents are also utilized. ${ }^{[10,11]}$

It is difficult to identify a definite time-point and causative event of the pseudoaneurysm in this patient's course. Her initial hematoma was most likely secondary to normal surgical bleeding, since it occurred within the usual timeframe and her anticoagulation had been normalized. Anticoagulation likely contributed to the acute large rebleed at 6 weeks, with rupture of a vessel that had sustained partial injury at the previous operation. It is also possible that the pseudoaneurysm developed after the second hematoma reoperation due to a missed transected arterial branch; this could occur with vessel contraction and a bloody operative field that obscured visualization. In addition, it is possible that the pseudoaneurysm developed later as a result of the aspiration procedure she underwent for presumed seroma. The aspiration procedure may have resulted in vessel trauma and pseudoaneurysm formation. Her history of anticoagulation is a known risk factor associated with pseudoaneurysm development.

Recurrent axillary hematomas are challenging entities to treat especially since it is difficult to identify causative bleeding vessels at the time of operation. For patients presenting with a firm mass after history of recurrent hematoma and who are symptomatic, fine needle aspiration is a reasonable approach. However, if the palpable mass persists after aspiration, or if the patient's medical comorbidities include management with anticoagulation, a diagnosis of pseudoaneurysm should 
be considered. Ultrasound is an ideal initial diagnostic test as it can distinguish liquified seroma from organized hematoma as well as identify arterial flow, as in this case. In patients who present with recurrent bleeding after mastectomy or axillary dissection, arterial imaging may demonstrate a hidden cause for the current bleeding, such as a pseudoaneurysm or arteriovenous fistula. Duplex arterial ultrasound may identify small pseudoaneurysms and allows immediate treatment using ultrasound-guided thrombin injection. A computed tomography angiography allows excellent image resolution of arterial anatomy and its relationship to adjacent structures. If a pseudoaneurysm is confirmed, treatment is recommended including the options of surgical exploration and arterial ligation, percutaneous thrombin injection and/or percutaneous arterial embolization.

\section{Conclusions}

Pseudoaneurysm formation can occur even after the most mundane of procedures such as seroma aspiration. Thus, it is important to keep these rare complications in mind when performing "small" procedures. Also, complicated patients are treated best with a multi-disciplinary team. The input from the breast imaging and surgical team experts led to appropriate next steps in the evaluation, management, and treatment of this patient. Finally, with increasing emphasis on minimally invasive treatments for managing complications, especially in patients with multiple co-morbidities, it should be remembered that an operation may be the quickest, least painful, and most effective approach.

\section{ACKNOWLEDGeMENTS}

The authors thank Marilyn Churchward for assistance with manuscript preparation.

\section{CONFLICTS OF INTEREST Disclosure}

The authors declare they have no conflict of interest.

\section{REFERENCES}

[1] Budd DC, Cochran RC, Sturtz DL, et al. Surgical morbidity after mastectomy operations. Am J Surg. 1978; 135: 218-220. http: //dx.doi.org/10.1016/0002-9610(78) 90103-4

[2] Lipshy KA, Neifeld JP, Boyle RM, et al. Complications of mastectomy and their relationship to biopsy technique. Ann Surg Oncol. 1996; 3: 290-294. http://dx.doi .org/10.1007/BF02306285

[3] Vitug AF, Newman LA. Complications in breast surgery. Surg Clin North Am. 2007; 87: 431-451. http://dx.doi.org/10.1016/j .suc.2007.01.005

[4] Barton MB, West CN, Liu IL, et al. Complications following bilateral prophylactic mastectomy. J Natl Cancer Inst Monogr. 2005: 61-66. http://dx.doi.org/10.1093/jncimonographs/lgi039

[5] Zion SM, Slezak JM, Sellers TA, et al. Reoperations after prophylactic mastectomy with or without implant reconstruction. Cancer. 2003; 98: 2152-2160. http://dx.doi.org/10.1002/cncr.11757

[6] Celik H, Yucel C, Oktar S, et al. Iatrogenic pseudoaneurysm of the superior thyroid artery: color Doppler ultrasonographic diagnosis and treatment approach. J Ultrasound Med. 2004; 23: 1675-1678.

[7] Dixon AM, Enion DS. Pseudoaneurysm of the breast: case study and review of literature. Br J Radiol. 2004; 77: 694-697. http: //dx.doi.org/10.1259/bjr/55440225

[8] World Bank. World Development Report 2000/2001: Attacking poverty. Washington, DC: World Bank; 2000. http://dx.doi.o rg/10.2214/AJR.05.1647

[9] Elford J, Burrell C, Roobottom C. Ultrasound guided percutaneous thrombin injection for the treatment of iatrogenic pseudoaneurysms. Heart. 1999; 82: 526-527. http://dx.doi.org/10.1136/hrt.8 2.4 .526

[10] Xenos ES, Freeman M, Stevens S, et al. Covered stents for injuries of subclavian and axillary arteries. J Vasc Surg. 2003; 38: 451-454 http://dx.doi.org/10.1016/S0741-5214 (03) 00553-6

[11] Castelli P, Caronno R, Piffaretti G, et al. Endovascular repair of traumatic injuries of the subclavian and axillary arteries. Injury. 2005; 36: 778-782. http://dx.doi.org/10.1016/j.injury.2004.1 2.046 